\title{
Superhydrophobic and Highly Oleophobic Zinc Sheet Surfaces Developed by a Simple Technique
}

\author{
Khedir R. Khedir ${ }^{1,2 *}$ \\ 1. Department of Applied Science, University of Arkansas at Little Rock, Little Rock, Arkansas, 72204, United States \\ 2. Department of Physics, College of Science, University of Duhok, Duhok 42001, Kurdistan Region, Iraq
}

\begin{abstract}
In this report, superhydrophobic and highly oleophobicmicro- nano-scale roughened Zn sheet surfaces were obtained. A simple and environmentally friendly technique of immersing $\mathrm{Zn}$ sheets in hot deionized water was used to grow $\mathrm{ZnO}$ nanorods. Variation of the immersion time had a significant impact on the evolution and formation of the nanorods. After their concealing with long chain fluorocarbon oligomers of $1 \mathrm{H}, 1 \mathrm{H}, 2 \mathrm{H}, 2 \mathrm{H}$-perfluorodecyltriethoxysilane (PFDTS), ZnO nanorod surfaces prepared at 60 min immersion time showed the highest wetting repellency properties toward water and peanut oil. However the surfaces yet to be able to super repel the two liquids. In order to improve the $\mathrm{Zn}$ sheet surfaces repellency toward liquids with various surface tensions, hierarchical structures with the combination of both micro channels covered-up with $\mathrm{ZnO}$ nanorods were developed. Simple technique of one-directional mechanical sanding was used to impart micro channeled structures onto Zn sheet surfaces and post immersion in hot de-ionized water for ZnO nanorods cover-up. The concealed micro-nano- combination structures with 1H, PFDTS oligomers showed superhydrophobic and highly oleophobic properties. Scanning electron microscopy (SEM), X-ray diffraction (XRD) and Photoluminescence (PL) techniques were used to characterize the morphology of the prepared surfaces.
\end{abstract}

Key words: Zn sheet, hot water, ZnO nanorods, hierarchical structures, superhydrophobic, oleophobic.

\section{Introduction}

In terms of structural properties, $\mathrm{ZnO}$ material can be formed in a wide range of morphological structures such as nanorods, nanowires, nanorings, nanobows, nanobelts, nanocomb, nanoflowers and nanopropeller [1]. This diversity in nanoscale morphological structure of $\mathrm{ZnO}$ has made it as a potential candidate for many technological applications. In addition to its wide potential application in electronics, it can also be used for the manipulation of solid surfaces wetting properties due to both significant increase in the surface area-to-volume ratio as well as transparency property of the material [2,3]. Solid surfaces with customized wetting properties could be used for a variety of applications such as self-cleaning, anti-fouling, anti-drag, water condensation, and water/oil separation. In addition, the efficiency of devices with such surface-wetting customized

\footnotetext{
*Corresponding author: Khedir R. Khedir, Ph.D., research
} fields: hydrophobicity, anti-icing and nanostructured surfaces. properties toward liquids is enhanced according to the existing environmental conditions.

Wetting properties of "smooth" solid surfaces is characterized primarily by the liquid droplet's contact angle, according to Young's model, while rested on the surface [4]. Contact angle of a liquid droplet is the angle between a tangent line to the droplet's side and the droplet's baseline. Its range is between $0^{\circ}-180^{\circ}$ depending on both solid surface's physical and chemical properties. A solid surface demonstrating a contact angle in the range of $10^{\circ}-90^{\circ}$ is known as a hydrophilic surface when the probe liquid is water and an oleophilic surface when the probe liquid is an organic liquid such as oil. In other words, the solid surface has the tendency toward wetting. For solid surfaces with a liquid contact angle in the range of $90^{\circ}-150^{\circ}$ are known as hydrophobic surfaces for water and oleophobic surfaces for organic liquids, demonstrating anti-wetting tendency. Solid surfaces with extreme liquid contact angles ranging from $0^{\circ}$ to 
$10^{\circ}$ are known as superhydrophilic (for water) and superoelophilic (for organic liquids), while the one with liquid contact angles ranging from $150^{\circ}$ to $180^{\circ}$ are known as superhydrophilic (for water) and superoelophilic (for organic liquids). Solid surfaces with both superhydrophobic and superoelophobic property are known as superamphiphobic surfaces that are useful for self-cleaning, anti-icing, anti-fouling and anti-drag applications [5].

Wetting properties of a solid surface can be manipulated by introducing physical and/or chemical modifications to the surface. Roughening magnifies wetting properties by the degree of increase in the actual surface area compared to the corresponding apparent area [6, 7]. In terms of chemical modifications, lowering the surface energy of a solid surface reduces its tendency to interact with liquids and consequent rise in the liquid droplet's contact angle. Nevertheless, the liquid's surface tension is also another vital parameter in determining the interaction mechanism between liquids and solid surfaces. Liquids with lower surface tensions such as organic liquids tend to interact more vigorously with solid surfaces, promoting lower contact angles. Therefore, developing rough solid surfaces that can strongly repel organic liquids such as oils is more challenging. In spite of chemical modification of rough solid surfaces with the lowest surface energy materials such as long chain fluorocarbons oligomers, carefully engineered rough structures with re-entrant propertyor combination of specific micro- nano-structured surfaces are demanded in order for the solid surface to strongly repel oils [8-10].

There have been reports on developing superhydrophobic $\mathrm{ZnO}$ nanostructured surfaces for anti-wetting applications [2, 3, 11-17]. Kwak et al. [14] reported superhydrophobic $\mathrm{ZnO}$ nanorods grown by wet-chemical technique after their surface modification with long chain hydrocarbon oligomers. It was also shown that the repellence tendency of the developed $\mathrm{ZnO}$ nanorod surfaces enhanced with the increase in hydrocarbon oligomers chain length. In another study by Gong et al. robust superhydrophobic $\mathrm{ZnO}$ nanowires were obtained after being grown on silicon micro pyramids structures without any post surface chemistry modification. Increase in the length of $\mathrm{ZnO}$ nanowires contributed in water droplet's transition from Wenzel state (wetting state) to Cassie state (non-wetting state). Wu et al. [2] also reported hybrid superhydrophobic $\mathrm{ZnO}$ surfaces grown by hydrothermal technique. The combination of $\mathrm{ZnO}$ nanorods topped with a porous zinc layer showed higher degree of stability.

Various techniques have been used to grow micro and/or nanostructured $\mathrm{ZnO}$ surfaces according to the suitability of $\mathrm{ZnO}$ developed nanostructures for a particular application [18-26]. The techniques used to grow $\mathrm{ZnO}$ nanostructures based on the required growth temperature and complexity can be divided into three main categories: First, high-temperature (> $500{ }^{\circ} \mathrm{C}$ ) thermal evaporation techniques such as metal-organic chemical vapor deposition, solid-vapor deposition, vapor-liquid-solid growth, and high pressure pulsed laser deposition. Second, moderate-temperature $\left(>100-500{ }^{\circ} \mathrm{C}\right)$ mechanisms such as: wet oxidation, glancing angle deposition. The third category is low temperature $\left(<100{ }^{\circ} \mathrm{C}\right)$, simple-solution based techniques such as; hydrothermal, sol-gel, electrochemical deposition, and wet-grinding. A specific growth technique can be used based on the demand for Zn oxide materials with a particular morphology and the choice of a substrate.

In an attempt to produce higher quality $\mathrm{ZnO}$ nanostructures using low temperature and simple-solution based techniques, Tan et al. [24] treated $\mathrm{Zn}$ foil with hot de-ionized water at $90{ }^{\circ} \mathrm{C}$ for relatively long periods of time ranging from 4 to $24 \mathrm{~h}$, in their study 2D nanosheets with different morphologies were obtained. In another study by the same group, $\mathrm{ZnO}$ nanowires were obtained after the polished Zn-foils pre-etched and then treated in hot de-ionized water for the same time periods. In both studies crystalline $\mathrm{ZnO}$ 
nanosheets and nanowire structures were reported.

In this study and encouraged by our previous studies on the treatment of metallic surfaces with hot de-ionized water for developing nanostructured metal oxide surfaces for anti-wetting property [27-29], the treatment of $\mathrm{Zn}$ sheet surfaces with hot water was carried out. Using this simple and environmentally friendly technique for relatively short durations ranging from 1 to $120 \mathrm{~min}$, crystalized $\mathrm{ZnO}$ nanorods with different shapes and morphological structures were imparted onto $\mathrm{Zn}$ sheet surfaces. In addition, hierarchical structures with the combination of micro channels covered-up with $\mathrm{ZnO}$ nanorods were also grown. Simple one-dimensional mechanical sanding was used to impart the micro channels onto the $\mathrm{Zn}$ sheet surfaces. The developed $\mathrm{Zn}$ sheet surfaces of micro, nano, and micro + nano were concealed with long chain fluorocarbon oligomers for anti-wetting property. The wetting characterization of the prepared Zn sheet surfaces was performed by using both water and peanut oil liquids. The morphology and the structure of the prepared surfaces were characterized using scanning electron microscopy (SEM), X-ray diffraction (XRD) and Photoluminescence (PL) techniques.

\section{Materials and Method}

\subsection{Surface Roughening}

First, commercially available $\mathrm{Zn}$ sheets with 1.0 $\mathrm{mm}$ thickness were cut into substrates with dimensions of $(2.5 \mathrm{~cm}$ X $2.5 \mathrm{~cm})$. For the removal of both the native oxide layer and environmental contamination, the substrates were polished using mechanical sanding utilizing two different ultrafine sandpapers of 2500 and 3000 grits, respectively. The flat-polished substrates were ultra-sonicated in acetone for $5 \mathrm{~min}$ and rinsed with de-ionized water. Next, the cleaned flat-polished zinc substrates were immediately immersed in hotde-ionized water $(\sim 100$ ${ }^{\circ} \mathrm{C}$ ) for the various durations of $1,5,20,60$ and 120 min. Hierarchical micro- nano-structured $\mathrm{Zn}$ sheet surfaces were obtained by first mechanically sanding the surfaces with 400 grit sandpaper for only 20 seconds. The sanding was carried out in one direction in order to impart micro channels to the $\mathrm{Zn}$ sheet surface. After cleaning, the developed $\mathrm{Zn}$ sheet surfaces with micro-channels were immersed in hot de-ionized water $\left(\sim 100^{\circ} \mathrm{C}\right)$ for a duration of $60 \mathrm{~min}$ to be covered-up with $\mathrm{ZnO}$ nanorods.

\subsection{Surface Characterization}

Morphology and geometrical dimensions of the formed micro-channels and $\mathrm{ZnO}$ nanorods were analyzed by top-view and cross-sectional scanning electron microscopy (SEM, JEOL 7000F) imaging. The crystal structure and orientation of the $\mathrm{ZnO}$ nanords were analyzed using X-ray diffraction (XRD, Bruker D8-Discover). Room temperature photoluminescence (PL) spectra of the $\mathrm{ZnO}$ nanostructured surfaces were obtained by using the He-Cd laser (IK Kimmon laser, IK5652R-G) with a wavelength of $325 \mathrm{~nm}$ and max power of $200 \mathrm{~m}$ was the excitation light source. After an exposure time of $15 \mathrm{~s}$, PL emission from the samples was collected by a detector (HORIBA JOBIN YVON, iHR320) with a vertical slit width of $0.4 \mathrm{~mm}$. PL measurements covered a spectral range from 150 to $1,230 \mathrm{~nm}$.

\subsection{Surface Chemistry Modification}

The roughened $\mathrm{Zn}$ sheet surfaces were immersed in hexane solution of $1 \mathrm{H}, 1 \mathrm{H}, 2 \mathrm{H}$, 2H-perfluorodecyltriethoxysilane (PFDTS) oligomers for 60 minutes. The chemically modified samples were rinsed with de-ionized water and placed inside oven under $90{ }^{\circ} \mathrm{C}$ for another $60 \mathrm{~min}$ in order for the reaction to be completed as well as removal of the unattached PFDTS oligomers. Finally, the surfaces were left in an ambient environment overnight.

\subsection{Wetting Analyses}

Wetting analyses of the physically and chemically modified Zn sheet surfaces was carried out using a 
VCA Optima goniometer. Liquid droplets of $5 \pm 1 \mu \mathrm{l}$ size of both water and peanut oil were gently dispensed on the surfaces. The Sessile Drop method was used to measure the CAs of the captured digital images.

\section{Results and Discussion}

Fig. 2 shows the top- and tilted-view SEM images of $\mathrm{Zn}$ sheet surfaces immersed in hot de-ionized water for durations of 1, 5, 20, 60 and $120 \mathrm{~min}$. A significant evolution in the morphology of nanostructures as the function of treatment time can be noticed. For the first min of immersion of $\mathrm{Zn}$ sheet substrates, individually separated tiny nanorods with an average size of less $20 \mathrm{~nm}$ are formed on the surface. After 5 min of immersion, densely packed and larger nanorods with a clear vertical preferential growth are developed. With the progress of the immersion time to reach $20 \mathrm{~min}$, dense and well vertically aligned individual nanorods are formed. According to the SEM images depicted in Fig. 2d, the $\mathrm{ZnO}$ nanorods possess two distinct diameters of around 20 and $50 \mathrm{~nm}$ both with a vertical height of slightly more than $100 \mathrm{~nm}$. After $60 \mathrm{~min}$ of immersion, nanorods with diameters of 50-75 nm and vertical heights of around $250 \mathrm{~nm}$ were formed which is twice the size of the nanorods formed after $20 \mathrm{~min}$ of immersion, Fig. 2e. Interestingly, the increase in immersion time of $\mathrm{Zn}$ sheet in hot water from 60 to 120 min showed decrease in the lateral size of nanorods with no significant variation in their vertical height. This indicates that the relatively well-aligned, individual and mono-dimension nanorods are formed after 60 min of immersion in hot water. In the studies carried out by Tan and his group [24] in treating unetched and etched $\mathrm{Zn}$ foil with hot water $\left(90^{\circ} \mathrm{C}\right)$, $\mathrm{ZnO}$ nanowires with $750 \mathrm{~nm}$ length for the etched $\mathrm{Zn}$

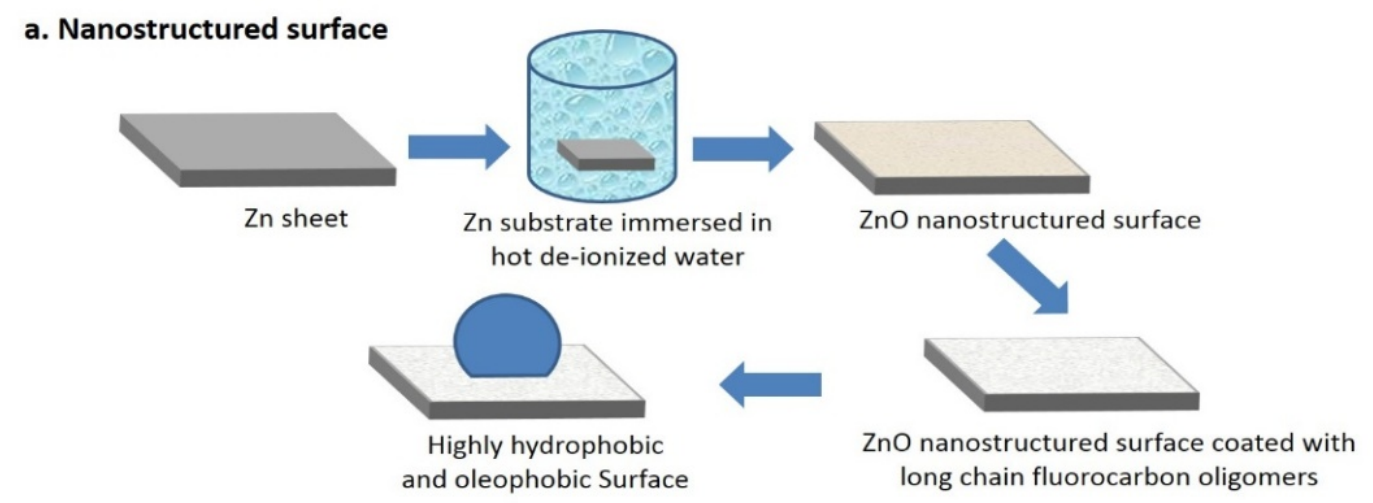

\section{b. Hierarchical Structured surface}

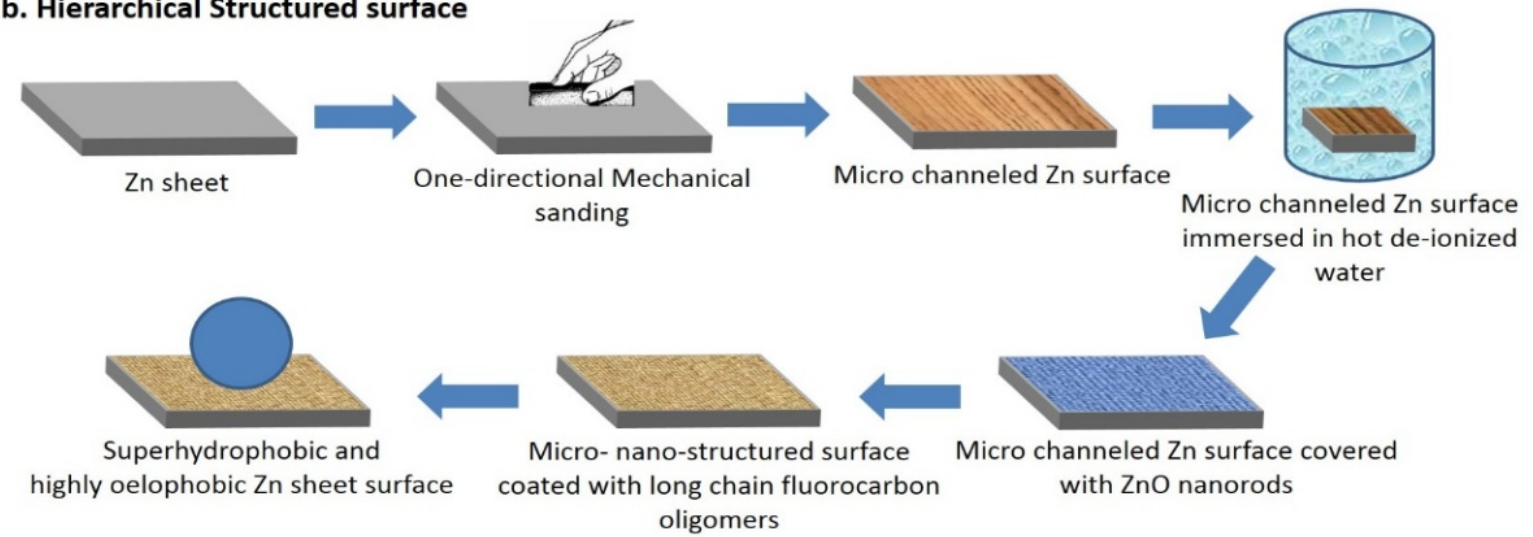

Fig. 1 Schematic representation of the experimental procedure (a) developing hydrophobic and oelophobic nanostructured Zn surface and (b) developing superhydrophobic and highly oleophobic hierarchical rough surface with micro channels covered with $\mathrm{ZnO}$ nanorods. 

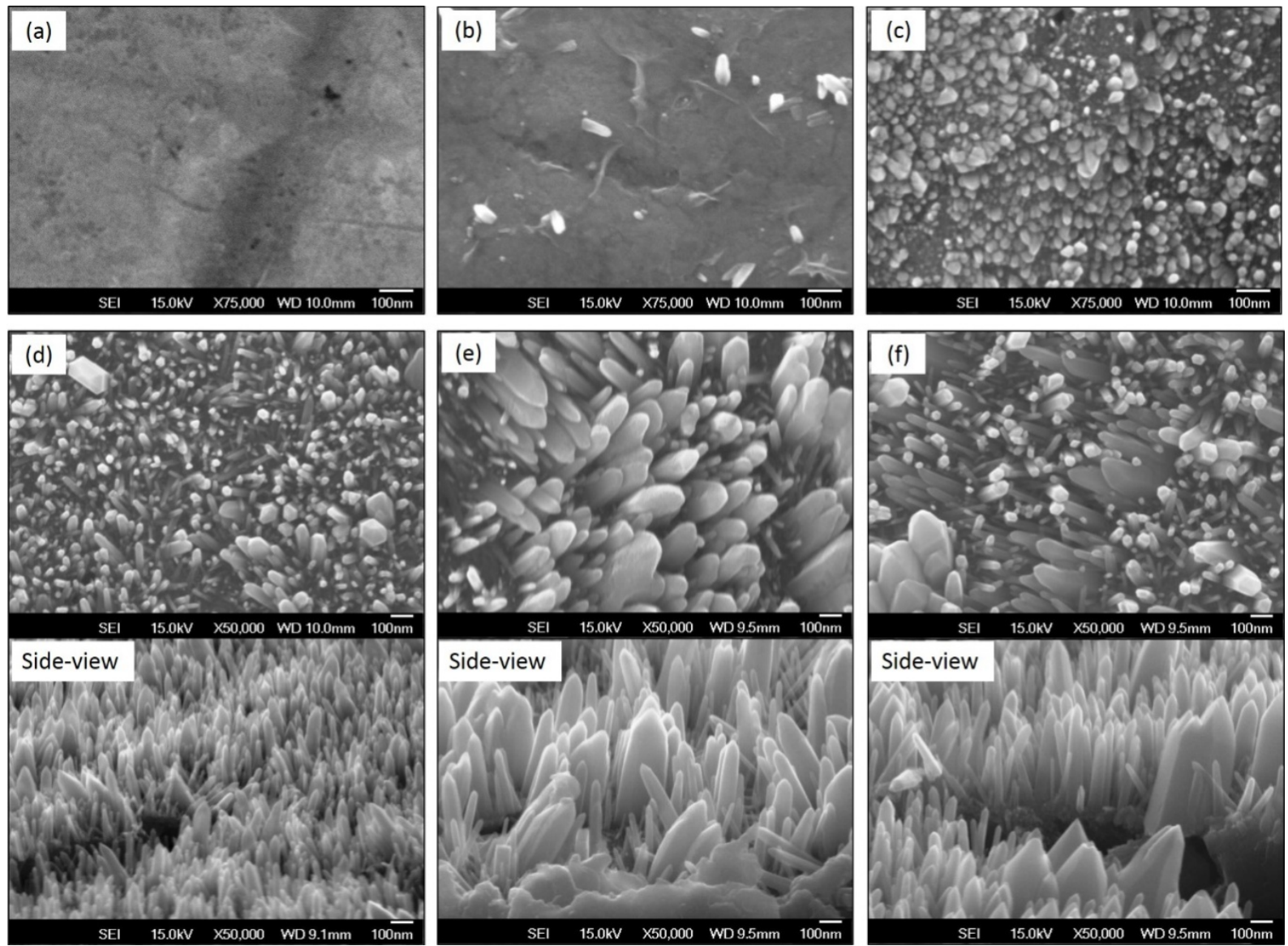

Fig. 2 SEM images of immersed zinc sheets in boiling DIW for various time periods of (a) untreated, (b) 1 min, (c) 5 min, (d) $20 \mathrm{~min}$, (e) $60 \mathrm{~min}$ and (f) $120 \mathrm{~min}$.

foil and dense nanosheets for the un-etched $\mathrm{Zn}$ foil were obtained.

The hexagonal shapes of the nanorods with multifacet tips is a primary manifestation of $\mathrm{ZnO}$ structures that have been widely reported in literature. To confirm that the obtained structures are $\mathrm{ZnO}$ with wurtezite structures, XRD analysis was carried out. The XRD peaks of the immersed Zn sheets in hot water for the different imerssion time periods are depicted in Fig. 3a. For the polished Zn sheet but untreated in hot water, peaks can be observed at $36.2^{\circ}$, $38.9^{\circ}, 43.2^{\circ}$ and $54.4^{\circ}$ correspond to the (002), (100), (101) and (102) orientations, respectively. For the Zn sheets treated in hot water for various durations, due to the strong peaks coming out from the $\mathrm{Zn}$ substrate only small peaks can be located that correspond to $\mathrm{ZnO}$ crystal structures. In addition, both $\mathrm{Zn}$ and $\mathrm{ZnO}$ materials share the same peak at $36.2^{\circ}$ but for the different orientations of (002) and (101), respectively $[24,30,31]$. With the progress in treatment time individual low intensity peaks from $\mathrm{ZnO}$ structures can be observed at different angles represnting the variation in the orientation of their crystal structure as the nanorods grow in size.

In order to be more certain that obtained nanorods are $\mathrm{ZnO}$ nanostructure and investigate their crystal quality and bandgap properties, room temperature PL analysis of the nanostructured surfaces was also performed. Fig. 3b shows the PL spectra of untreated $\mathrm{Zn}$ sheet and $\mathrm{Zn}$ sheet surfaces treated in hot de-ionized water for various time periods of $1,5,20$, 60 , and $120 \mathrm{~min}$. In general, a main intense band of UV emission is observed at $377-391 \mathrm{~nm}$, associated with the energy band gaps of 3.28-3.17 eV. The plot shows that the band intensifies with the increase in treatment time, which is consistent with the morphological 
(a)

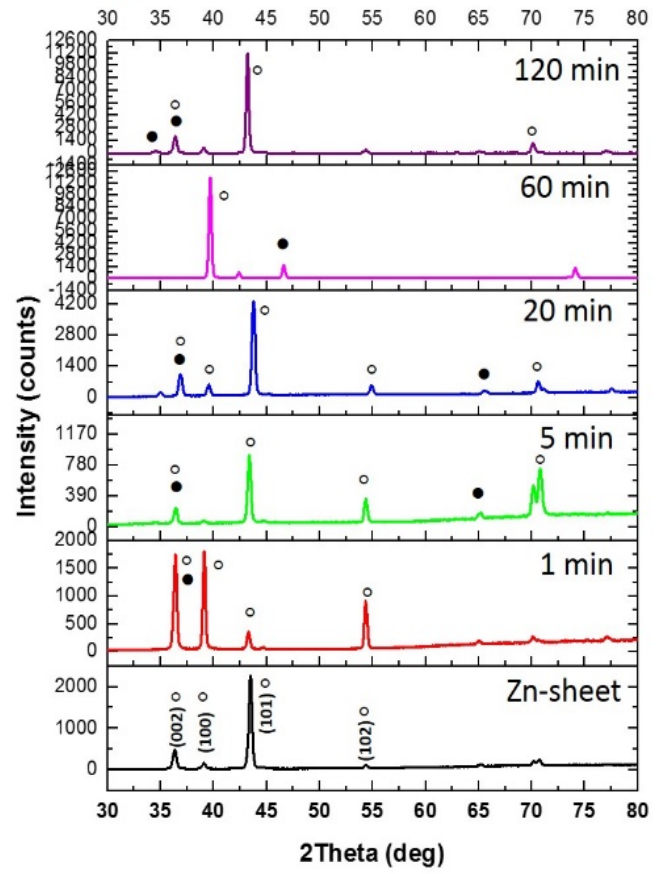

(b)

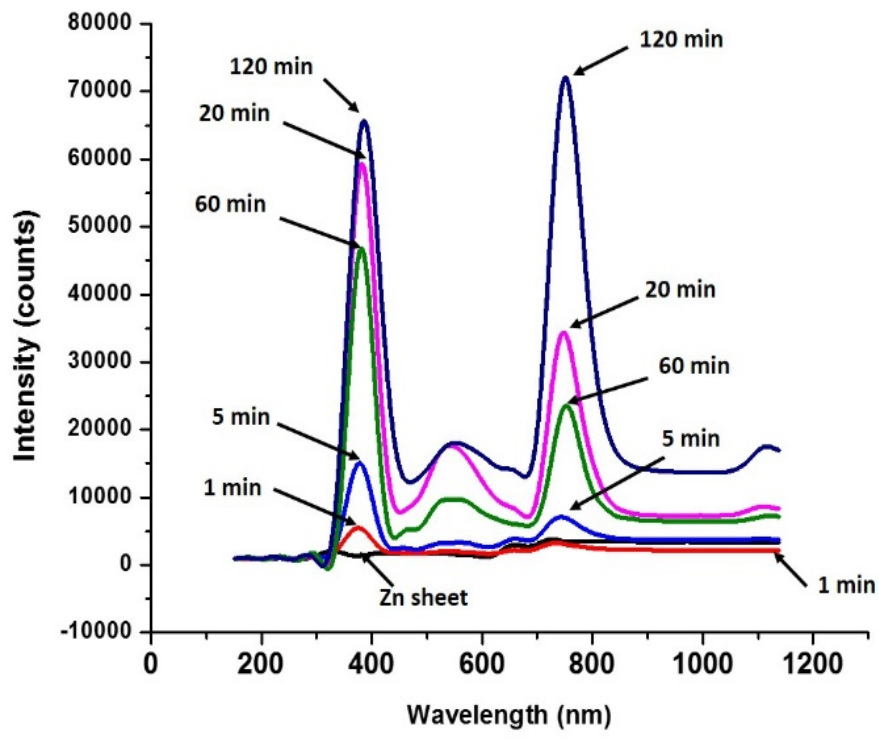

Fig. 3 XRD and photoluminscence spectra of the untreated and treated $\mathrm{Zn}$ sheet srfaces in hot de-ionized water for different time periods of $1,5,20,60$ and $120 \mathrm{~min}$.

growth of $\mathrm{ZnO}$ nanostructures. However, $\mathrm{ZnO}$ nanorods obtained after 20 min of immersion showed more intense peak compared to the $\mathrm{ZnO}$ obtained after 60 min of immersion, which indicates higher quality $\mathrm{ZnO}$ nanorods after $20 \mathrm{~min}$ immersion compared to the one obtained after $60 \mathrm{~min}$ immersion. The energy band gap of the obtained $\mathrm{ZnO}$ nanorods is relatively consistent with the reported energy band gaps of $\mathrm{ZnO}$ nanorod and nanowire structures. The second intense band is observed at infrared region with a wavelength of $743 \mathrm{~nm}$, associated to narrow band gap of 1.66 $\mathrm{eV}$. The third but weakest band is located in the green region at 545-521 $\mathrm{nm}$ associated to energy band gap of 2.27-2.38 eV. According to literature, the band observed in the green region is believed to be due to both defects in the structure of the obtained $\mathrm{ZnO}$ nanorods and/or size effects of the nanorods [31].

The formation mechanism of the $\mathrm{ZnO}$ nanorods formed on the surface of $\mathrm{Zn}$ sheets after their immersion in hot de-ionized water can be explained in the context of the both hydrothermal and wet oxidation techniques. In the hydrothermal technique double-positive $\mathrm{Zn}$ ions $\left(\mathrm{Zn}^{2+}\right)$ are generatedas the result of chemcial reactionsbetween the mixed solvents. With time progress and sustained heat (90 $\left.{ }^{\circ} \mathrm{C}\right), \mathrm{Zn}(\mathrm{OH})_{2}$ moleculesare generated after $\mathrm{Zn}^{2+}$ ion hydrolyses combined with two negative hydroxyl groups $\left(2 \mathrm{OH}^{-}\right)$. Finally and due to the saturation of the $\mathrm{Zn}$ sheet surfaces with $\mathrm{Zn}(\mathrm{OH})_{2}$ molecules, $\mathrm{ZnO}$ structures began to nucleate as the intial stage of $\mathrm{ZnO}$ nanostructure's growth. In our case of hot water treament, no chemical solvents are involved to obtain the $\mathrm{Zn}^{2+}$ ions, so we believe that the source of $\mathrm{Zn}^{2+}$ ions on the surface of $\mathrm{Zn}$ sheet is the diffusion of $\mathrm{Zn}$ atoms across the surface. This is similar to the case of wet oxidation of $\mathrm{Zn}$ films for developing $\mathrm{ZnO}$ nanostructures but under much higher temperature approaching $\mathrm{Zn}$ melting point $\left(420^{\circ} \mathrm{C}\right)$. Furthermore, the source of providng the $\mathrm{OH}^{-}$groups could be the interaction between both $\mathrm{Zn}$ surfaces and $\mathrm{H}_{2} \mathrm{O}$ molecules at which the surface of the $\mathrm{Zn}$ metallic sheet acts as a catalyst to break down water molecules into $\mathrm{OH}^{-}$and $\mathrm{H}^{+}$. Each of a two hydroxl groups $\left(2 \mathrm{OH}^{-}\right)$bonds into a diffused zinc ion $\left(\mathrm{Zn}^{2+}\right)$ and 
forms $\mathrm{Zn}(\mathrm{OH})_{2}$ molecules. Next, nucleation of $\mathrm{ZnO}$ nanostructures starts to initiate due to the saturation of the surface with $\mathrm{Zn}^{2+}$ ions. The formed $\mathrm{ZnO}$ nucleation sites promote the formation of $\mathrm{ZnO}$ nanostructures by a sequence stacking of $\mathrm{Zn}^{2+}$ and $\mathrm{O}^{2-}$ to form nanorods. The folowing two equations showcase the formation process of $\mathrm{ZnO}$ nanorods [32, 33].

$$
\begin{gathered}
\mathrm{Zn}^{2+}+2 \mathrm{OH}^{-} \leftrightarrow \mathrm{Zn}(\mathrm{OH})_{2} \\
\mathrm{Zn}(\mathrm{OH})_{2} \leftrightarrow \mathrm{ZnO}+\mathrm{H}_{2} \mathrm{O}
\end{gathered}
$$

In order to develop hierarchical structures with both micro- nano- combination, simple mechanical sanding technique was combined with hot water treatment technique. Fig. 4 shows the SEM images for the developed hierarchical structures of $\mathrm{Zn}$ micro channels uniformly roofed with $\mathrm{ZnO}$ nanorods. The micro channels were imparted via one-directional mechanical sanding using 400 grit and post immersion in hot water for the duration of $60 \mathrm{~min}$. As shown in the SEM images, the channels have dimensions with few microns and a relatively unequal distance from each other ranging from few microns to more than 10 microns. For more uniform micro channels in terms of dimensions and separation distance, a more sophisticated mechanism for mechanical sanding is required. In spite of that, the channels are uniformely roofed with $\mathrm{ZnO}$ nanorods with dimensions and shapes close to the ones obtained on smooth $\mathrm{Zn}$ sheet shown in Fig. 2e. Interestingly, the inner sides of micro channels are also uniformly concealed with $\mathrm{ZnO}$ nanorods, as demonstrated in the magnified SEM images of Fig. 4, which could enhance the re-entrant property of the hierarchical structures. In addition to both roughness and low surface energy, the property is required for the engineered surfaces to strongly repel low surface tension liquids.

In order to impart liquid repellency property toward water and oils onto the prepared Zn sheet surfaces of nanostructures, the surfaces were concealed with long chain fluorocarbon oligomers of PFDTS. Wetting

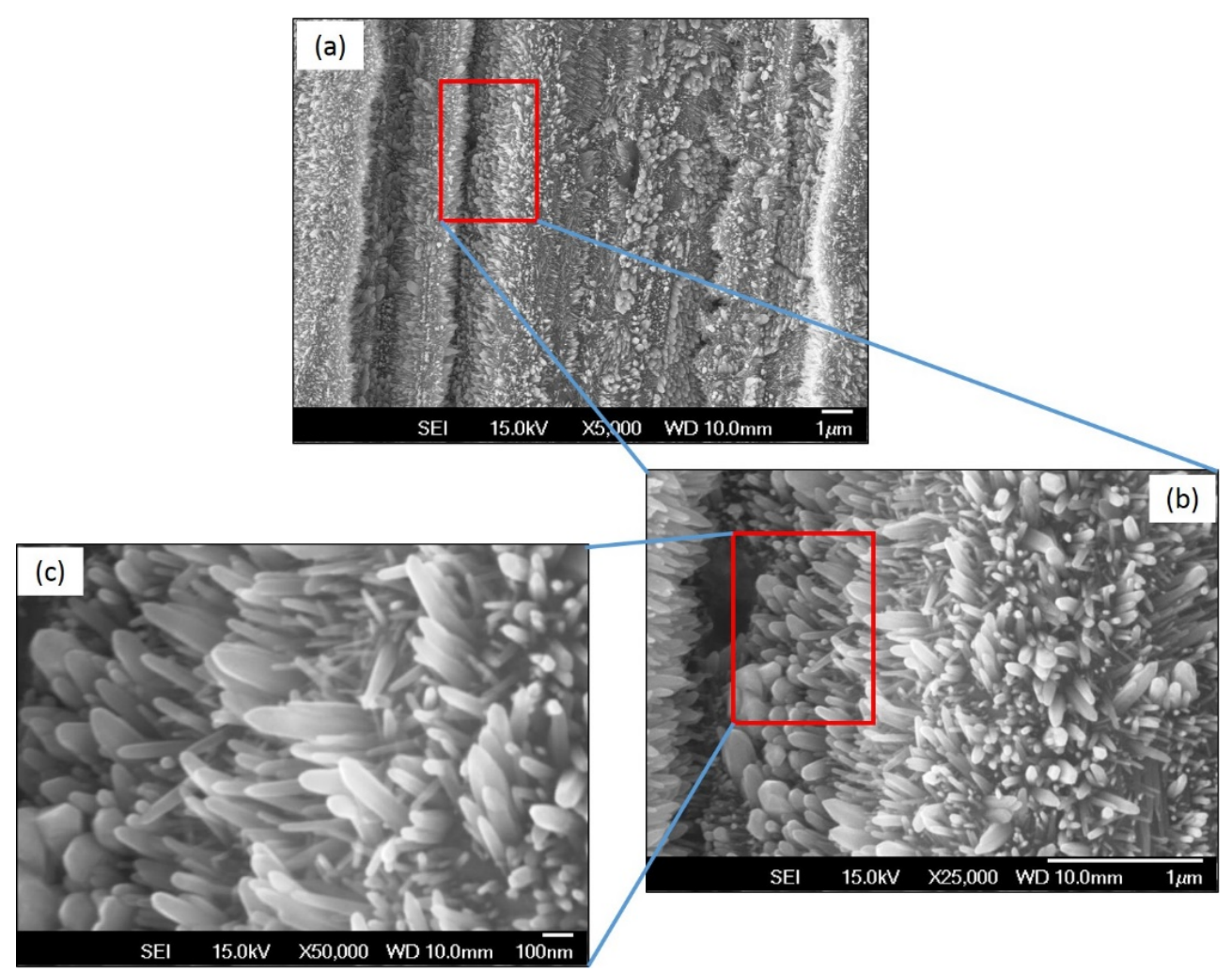

Fig. 4 SEM images of Zn micro channels covered with ZnO nanorods obtained by combination of both mechanical sanding with $\mathbf{4 0 0}$ grit sandpaper and post treatement in hot water for $60 \mathrm{~min}$. 
characterization of the $\mathrm{Zn}$ sheet surfaces with $\mathrm{ZnO}$ nanorod structures after being concealed with PFDTS oligomers is depicted in Fig. 5. A clear trend of increase in the values of both water and peanut oil contact angles is observed with the increase in the immersion time of the surfaces in hot water for the first $60 \mathrm{~min}$ time period. However, for the surfaces treated for 120 min duration, lower contact angles for both liquids were observed compared to the $60 \mathrm{~min}$ immersion time. This can be qualitatively attributed to the fact that $\mathrm{ZnO}$ nanorods obtained for $60 \mathrm{~min}$ immersion time, as shown in the SEM images of Fig. 2e, are better individually-aligned with larger lateral and vertical dimensions. All these parameters promote significant increase in the apparent surface area of the obtained rough surfaces which in turns magnifies the liquid repellency of the surfaces. The surface observed a water contact angle of $141^{\circ}$ and a peanut oil contact angle of $100^{\circ}$, see Fig. 5b. The wetting repellency property of the prepared surfaces is significantly enhanced, but hasn't reached super repellency at least toward high surface tension water.

In order to enhance the wetting repellency of the $\mathrm{Zn}$ sheet surfaces toward liquids to reach super-repellency, a combination of Zn micro channels covered-up with $\mathrm{ZnO}$ nanorods were also developed.
The wetting properties of the hierarchical structured Zn sheet surfaces after being concealed with long chain fluorocarbon oligomers of PFDTS were also measured. The micro channel structures covered-up with nanorods observed water contact angle of $157^{\circ}$ and peanut oil contact angle of $118^{\circ}$ as shown in the Fig. 6 . The figure also illustrates the effect of different ugh scales of micro, nano and the combination of micro/nanostructures imparted onto $\mathrm{Zn}$ sheet surfaces, after being concealed with long chain fluorocarbon oligomers of PFDTS, on their wetting repellency properties. In terms of high surface tension water, the wetting repellency of the prepared $\mathrm{Zn}$ sheet surfaces monotonically increased starting from contact angle of $127^{\circ}$ for micro-rough structures, $141^{\circ}$ for nano-rough structures, and reaching superhydrophobicity with contact angle of $157^{\circ}$ for combined micro-nano rough structures. For the low surface tension liquid of peanut oil, the same trend of enhancement in the repellency properties of the prepared surfaces can be noticed but with relatively lower contact angles. It is concluded that the Hierarchical structure design significantly enhanced the repellency of the $\mathrm{Zn}$ sheet surfaces toward high surface tension liquids such as water and with a lower rate toward low surface tension liquids as well. (a)

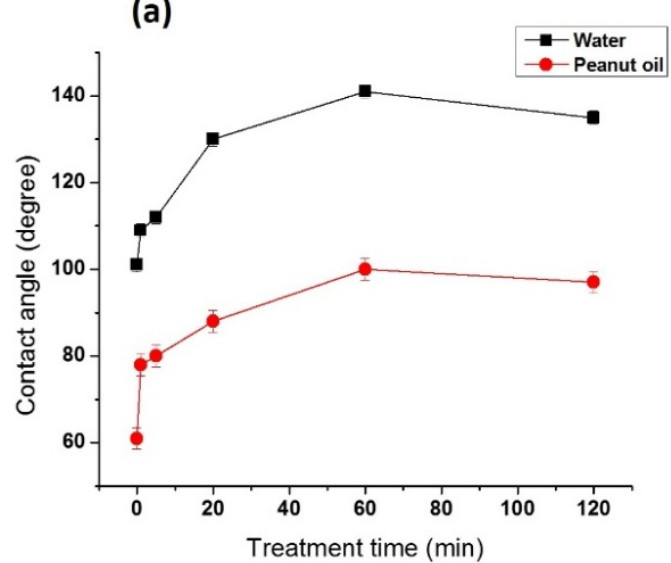

(b)

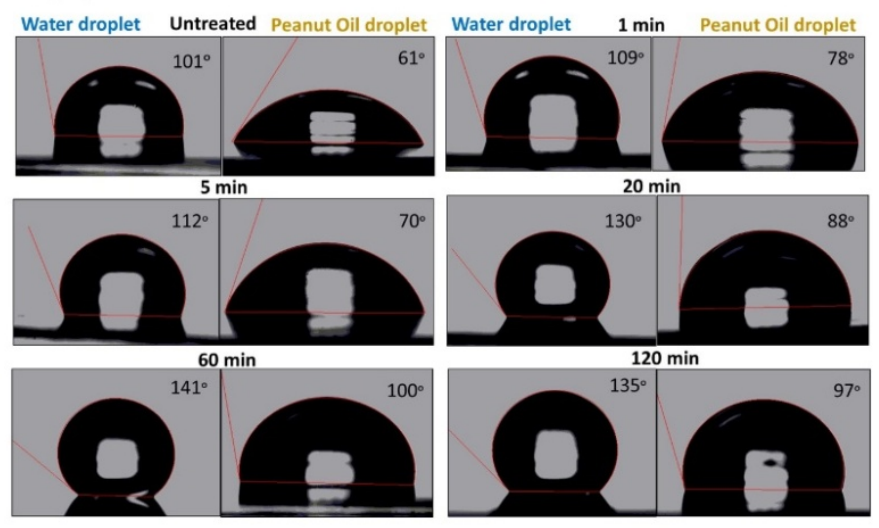

Fig. 5 (a) Water and Peanut oil contact angles versus treatment time of Zn sheet surfaces with hot de-ionized water ( 100 ${ }^{\circ} \mathrm{C}$ ) and post concealing with long chain fluorocarbon oligomers, (b) digital images of both water and peanut oil droplets. 
(a)

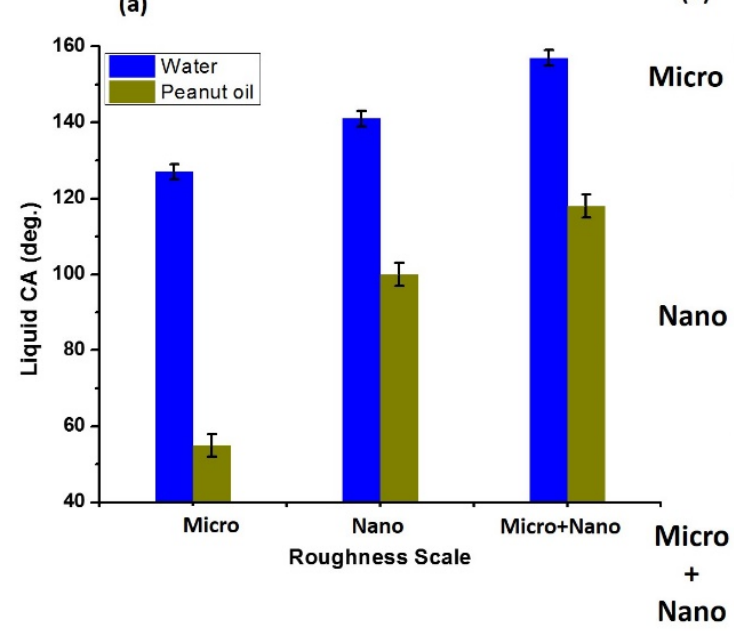

(b)
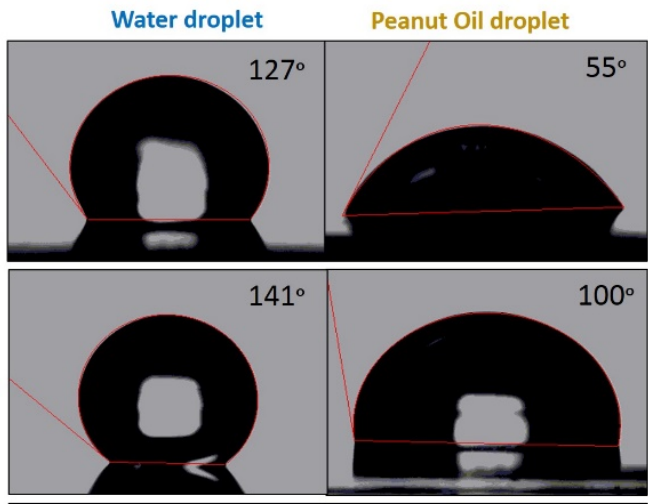

$157^{\circ}$

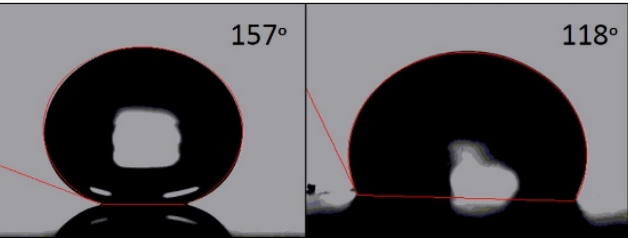

Fig. 6 (a) Water and Peanut oil CAs versus the three different roughness scales of the developed Zn sheet surfaces concealed with long chain fluorocarbon of PFDTS oligomers, (b) digital images of both water and peanut oil droplets while rested on those surfaces.

\section{Conclusions}

Simple and eco-friendly technique of mechanical sanding combined with hot water treatment is used to develop highly liquid repellent $\mathrm{Zn}$ sheet surfaces toward both high and low surface tension liquids after lowering their surface energy with long chain fluorocarbon oligomers. Nano-scalerough surfaces with various dimensions and density formed by hot water treatment significantly enhanced the $\mathrm{Zn}$ sheet surfaces liquid repellency properties. However, super repellent $\mathrm{Zn}$ sheet surfaces toward high surface tension liquids and high repellency toward organic liquids were only obtained after combining both mechanical sanding and hot water treatment for developed micro- nano-structured combination and its post concealing with long chain fluorocarbon oligomers.

\section{Acknowledgements}

The author would like to thank Dr. Tansel Karabacac's group from Department of Physics and Astrnonmy at University of Arkansas at Little Rock (UALR), USA,for their help in sample characterization. Also the help from the UALR Center for Integrative Nanotechnology Sciences for SEM and XRD analyses is highly appreciated.

\section{References}

[1] Xu, S. and Wang, Z. L. 2011. "One-Dimensional ZnO Nanostructures: Solution Growth and Functional Properties.” Nano Res. 4: 1013-98.

[2] Wu, J., Yang, X., Lei, W., Xia, J. and Wang, B. 2013. "Superhydrophobic Zinc Oxide Film: Effect of Hybrid Nanostructure on Hydrophobicity and Wetting Stability." Micro and Nano Lett. 8: 271-3.

[3] Zheng, L., Li, Z., Bourdo, S., Saini, V., Ryerson, C. and Biris, A. S. 2011. "Hierarchical ZnO Structure with Superhydrophobicity and High Adhesion.” Chem. Phys. Chem. 2011: 2412-4.

[4] Young, T. 1805. "An Essay on the Cohesion of Fluids.” Philos. Trans. Roy. Soc. London. 95: 65-87.

[5] Bhushan, B. and Yong, C. J. 2011. "Natural and Biomimetic Artificial Surfaces for Superhydrophobicity, Self-Cleaning, Low Adhesion, and Drag Reduction.” Prog. Mater. Sci. 56: 1-108.

[6] Wenzel, R. N. 1936. "Resistance of Solid Surface to Wetting by Water.” Ind. Eng. Chem. 28: 988-94.

[7] Cassie, A. B. D. and Baxter, S. 1944. "Wettability of Porous Surfaces.” Trans. Faraday Soc. 40: 546-51.

[8] Deng, X., Mammen, L., Butt, H.-J. and Vollmer, D. 2012. "Candle Soot as a Template for a Transparent Robust Superamphiphobic Coating.” Science 335: 67-70.

[9] Lafuma, A. and Quere, D. 2003. "Superhydrophobic States." Nat. Mater. 2: 457-60. 
[10] Khedir, K. R., Kannarpady, G. K., Ishihara, H., Woo, J., Ryerson, C. and Biris, A. S. 2011. "Design and Fabrication of Teflon-Coated Tungsten Nanorods for Tunable Hydrophobicity.” Langmuir 27: 4661-8.

[11] Wang, B., Zhao, Y. and Yu, T. 2011. "Fabrication of Novel Superhydrophobic Surfaces and Droplet Bouncing Behavior - Part 2: Water Droplet Impact Experiment on Superhydrophobic Surfaces Constructed Using ZnO Nanoparticles.” J. Adhes. Sci. Technol. 25: 93-108.

[12] Liu, C., Zhang, Q., Li, T. and Li, Z. 2010. "Superoleophobic and Superhydrophobic Surfaces from Microtextured ZnO-Based Surfaces on Si Substrate.” Key Eng. Mat. 434-435: 538-41.

[13] Wang, B., Feng, J., Zhao, Y. and Yu, T. X. 2010. "Fabrication of Novel Superhydrophobic Surfaces and Water Droplet Bouncing Behavior - Part 1: Stable ZnO-PDMS Superhydrophobic Surface with Low Hysteresis Constructed Using ZnO Nanoparticles.” J. Adhes. Sci. Technol. 24: 2693-705.

[14] Kwak, G., Seol, M., Tak, Y. and Yong, K. 2009. "Superhydrophobic ZnO Nanowire Surface: Chemical Modification and Effects of UV Irradiation.” J. Phys. Chem. C 113: 12085-9.

[15] Sakai, M., Kono, H., Nakajima, A., Zhang, X., Sakai, H. and Abe, M. et al. 2009. "Sliding of Water Droplets on the Superhydrophobic Surface with ZnO Nanorods." Langmuir 25: 14182-6.

[16] Shaik, U. P., Kshirsagar, S., Krishna, M. G., Tewari, S. P., Purkayastha, D. D. and Madhurima, V. 2012. "Growth of Superhydrophobic Zinc Oxide Nanowire Thin Films.” Mater. Lett. 75: 51-3.

[17] Wu, J., Chen, J., Xia, J., Lei, W. and Wang, B.-P. 2013. "A Brief Review on Bioinspired ZnO Superhydrophobic Surfaces: Theory, Synthesis, and Applications.” Adv. Mate. Sci. Eng. 2013: 232681.

[18] Al-Owais, A. 2013. "Synthesis and Magnetic Properties of Hexagonally Packed ZnO Nanorods.” Arabian Journal of Chemistry 6: 229-34.

[19] Chen, H., Lian, H. Hung,, S. and Wang, C. 2013. "Epitaxial Growth of Self-Ordered ZnO Nanostructures on Sapphire Substrates by Seed-Assisted Hydrothermal Growth.” J. Cryst. Growth 362: 231-4.

[20] Kale, R. B. and Lu, S. 2013. "Hydrothermal Growth and Characterizations of Dandelion-like ZnO Nanostructures." J. Alloy. Compod. 579: 444-9.

[21] Lin, W., Shih, C., Wu, C. and Seshia, A. A. 2013. "Synthesis of Zinc Oxide Nanostructures by Microheaters in the Ambient Environment.” IEEE Trans. Nanotech. 12: 21-8.
[22] Noothongkaew, S., Pukird, S., Sukkabot, W., Kasemporn, B., Songsiririttikul, P. and An, K. 2013. "Zinc Oxide Nanostructures Synthesized by Thermal Oxidation of Zinc Powder on Si Substrate.” Appl. Mech. Mater. 328: 710-4.

[23] Rivera, A., Zeller, J., Sood, A. and Anwar, M. 2013. “A Comparison of ZnO Nanowires and Nanorods Grown Using MOCVD and Hydrothermal Processes.” J Electron Mater. 42: 894-900.

[24] Wai, K. T., Abdul Razak, K., Lockman, Z., Kawamura, G., Muto, H. and Matsuda, A. 2013. "Formation of Highly Crystallized ZnO Nanostructures by Hot-Water Treatment of Etched Zn Foils.” Mater. Lett. 91: 111-4.

[25] Cui, J. 2012. “Zinc Oxide Nanowires.” Mater. Charact. 64: 43-52.

[26] Kim, J.-H., Kim, K.-W., Ryu, K.-S. and Cho, K.-K. 2012. "Fabrication of $\mathrm{ZnO}$ Nanorods by Electrochemical Deposition Process and its Photovoltaic Properties." Mater. Technol. 27: 18-20.

[27] Khedir, K. R., Saifaldeen, Z. S., Demirkan, T. M., Al-Hilo, A. A., Brozak, M. P. and Karabacak, T. 2014. "Robust Superamphiphobic Nanoscale Copper Sheet Surfaces Produced by a Simple and Environmentally Friendly Technique.” Adv. Eng. Mater. 17: 982-9.

[28] Saifaldeen, Z. S., Khedir, K. R., Cansizoglu, M. F., Demirkan, T. and Karabacak, T. 2014. "Superamphiphobic Aluminum Alloy Surfaces with Micro and Nanoscale Roughness Produced by a Simple and Environmentally Friendly Technique.” J. Mater. Sci. 49: 1839-53.

[29] Saifaldeen, Z. S., Khedir, K. R., Camci, M. T., Ucar, A. and Suzer, S. et al. 2016. "The Effect of Polar End of Long-Chain Fluorocarbon Oligomers in Promoting the Superamphiphobic Property over Multi-Scale Rough Al Alloy Surfaces.” Appl. Surf. Sci. 379: 55-65.

[30] Tan, W. K., Razak, K. A., Lockman, Z., Kawamura, G., Muto, H. and Matsuda, A. 2013. "Optical Properties of Two-Dimensional ZnO Nanosheets Formed by Hot-Water Treatment of Zn Foils.” Solid State Commun. 162: 43-7.

[31] Qiu, Z., Nadamura, Y. and Ishiguro, T. 2010. "Formation of Zinc Oxide Film by Boiling Metallic Zinc Film in Ultrapure Water.” Thin Solid Films 518: 5912-5.

[32] Ohmura, S., Shimojo, F., Kalia, R. K., Kunaseth, M., Nakano, A. and Vashishta, P. 2011. "Reaction of Aluminum Clusters with Water.” J. Chem. Phys. 134: 244702.

[33] Nie, H.-Q., Zhang, S.-S., Schoenitz, M. and Dreizin, E. L. 2013. "Reaction Interface between Aluminum and Water." Int. J. Hydrogen Energy 38: 11222-32. 